\title{
Paradoxical hemopericardium in type B acute aortic dissection
}

Hisato Takagi, MD, PhD, Hideaki Manabe, MD, Seishiro Sekino, MD,

Takayoshi Kato, MD, Yukihiro Matsuno, MD, PhD, and

Takuya Umemoto, MD, PhD, Shizuoka, Japan

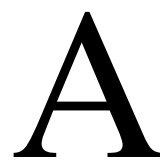

lthough hemopericardium often occurs in Stanford type A acute aortic dissection or intramural hematoma, any reports of this in type B dissection have never been found in the literature. ${ }^{1}$ We here describe the first case of cardiac tamponade caused by hemopericardium in type B acute thrombosed aortic dissection.

\section{Clinical Summary}

A 71-year-old man who had been medicated for hypertension and gout presented with acute back pain and subsequent chest pain. Pallor, perspiration, neck vein distention, tachycardia (108 beats/min), hypotension $(93 / 58 \mathrm{~mm} \mathrm{Hg})$, and anuria were rec-

\footnotetext{
From the Department of Cardiovascular Surgery, Sizuoka Medical Center, Shizuoka, Japan.

Received for publication July 14, 2004; revisions received Aug 7, 2004; accepted for publication Aug 10, 2004.

Address for reprints: Hisato Takagi, MD, PhD, Department of Cardiovascular Surgery, Sizuoka Medical Center, 762-1 Nagasawa, Shimizu-cho, Sunto-gun, Shizuoka 411-8611, Japan (E-mail: kfgth973@ybb.ne.jp).

J Thorac Cardiovasc Surg 2005;129:939-40

0022-5223/\$30.00

Copyright () 2005 by The American Association for Thoracic Surgery doi:10.1016/j.jtcvs.2004.08.011
}

ognized. Moderate pericardial effusion without aortic valve regurgitation was disclosed on echocardiography. Computed tomographic scanning revealed dissection in the descending thoracic aorta with a noncontrasted false lumen (Figure 1), a slightly dilated ascending aorta of $4 \mathrm{~cm}$ in maximum diameter without dissection or intramural hematoma (Figure 1, A), and pericardial effusion of $2.5 \mathrm{~cm}$ in maximum thickness (Figure 1, $B$ ). The patient was given a diagnosis of Stanford type B (DeBakey type III) acute aortic dissection with cardiac tamponade. Pericardiocentesis was urgently performed, and approximately $400 \mathrm{~mL}$ of bloody fluid was drained. The clinical conditions were remarkably improved, and intravenous antihypertensive treatment for type B acute aortic dissection was initiated immediately after the drainage.

\section{Discussion}

According to Motoyoshi and associates, ${ }^{2}$ the incidences of preoperative pericardial effusion in patients with acute Stanford type A aortic intramural hematoma and dissection are $22 \%$ (8/36 patients) and $44 \%$ (22/50 patients), respectively. Although the former is significantly lower than the latter, early operation is preferred for
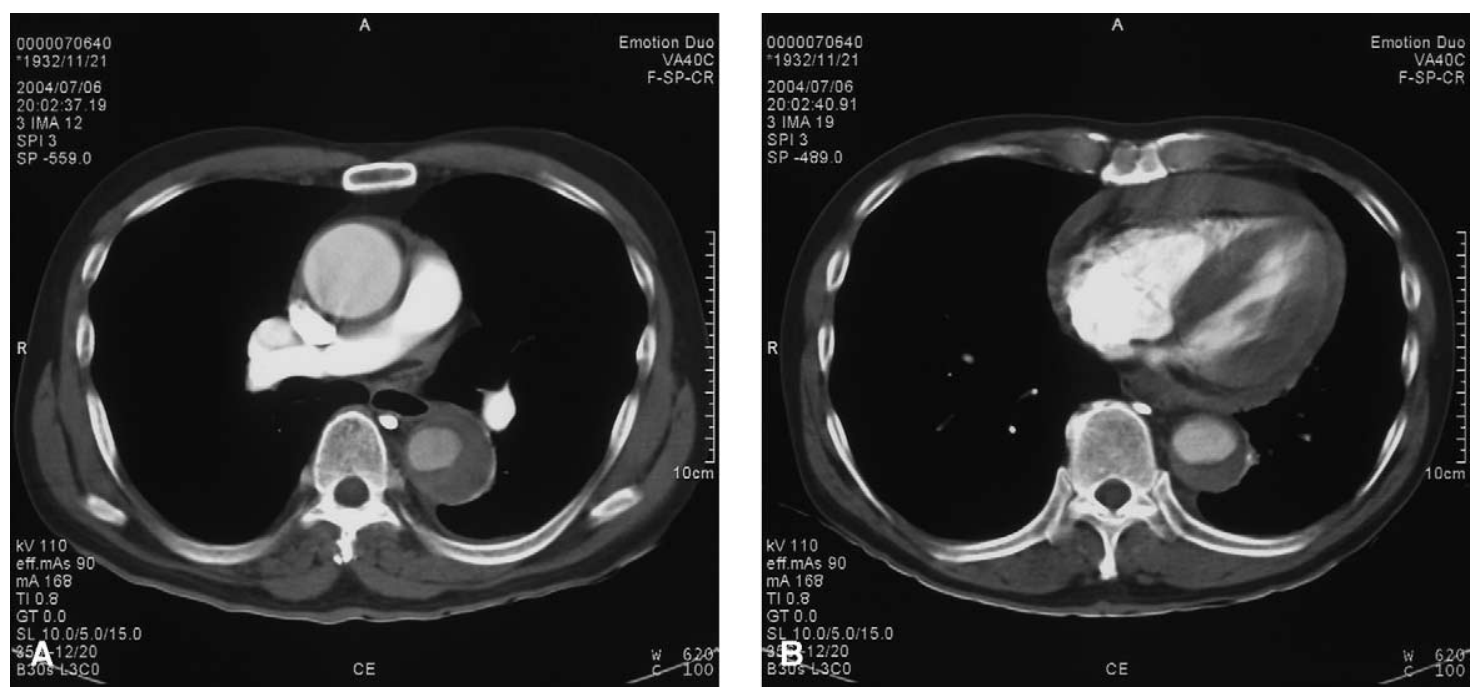

Figure 1. Computed tomographic scanning revealed dissection in the descending thoracic aorta with noncontrasted false lumen (A and B), a slightly dilated ascending aorta of $4 \mathrm{~cm}$ in maximum diameter without dissection or intramural hematoma (A), and pericardial effusion of $2.5 \mathrm{~cm}$ in maximum thickness (B). 
intramural hematoma involving the ascending aorta with cardiac tamponade. On the contrary, pericardial effusion is considered not to occur theoretically in type B acute aortic dissection or intramural hematoma because the ascending aorta is not involved. ${ }^{1}$

The diagnosis of acute type A aortic dissection is predominantly based on the demonstration of an intimal tear or a dissection membrane. Attenhofer and collaborators, ${ }^{3}$ however, have described another pathogenetic mechanism in a patient with the typical features of acute aortic dissection with pericardial tamponade and a giant aneurysm of the ascending aorta, where no dissection membrane, rupture site, or intimal tear could be demonstrated by transesophageal echocardiography, intraoperative examination, or histologic examination. The histologic work-up showed an extreme form of cystic medial necrosis, with intramural hemorrhages consistent with a leaking aneurysm. In the setting of an extreme medial necrosis, blood can leak through the aortic wall, causing intramural hemorrhages with intimal leaks invisible to the surgeon's or echocardiographer's eye. In the present case involving type B acute aortic dissection, hemopericardium resulting in cardiac tamponade occurred paradoxically. Intramural hemorrhage invisible to computed tomographic scanning caused by cystic medial necrosis, which could cause the dissection, might have existed.

Cardiac tamponade frequently complicates acute proximal aortic dissection and is one of the most common causes of death from aortic dissection. In Isselbacher and colleagues' study of 10 patients who were given diagnoses of both aortic dissection and cardiac tamponade, ${ }^{4} 3$ died from electromechanical dissociation immediately on presentation; 3 other deaths all occurred shortly after successful pericardiocentesis, a procedure undertaken to stabilize the patients. The observations do raise the possibility that in patients with cardiac tamponade complicating aortic dissection, pericardiocentesis could be harmful rather than beneficial. Because neither acute aortic dissection nor intramural hematoma involved the ascending aorta in the present case, we performed pericardiocentesis to relieve the cardiac tamponade, and this fortunately stabilized the patient's condition.

\section{References}

1. Miller DC, Stinson EB, Oyer PE, Rossiter SJ, Reitz BA, Griepp RB, et al. Operative treatment of aortic dissections. Experience with 125 patients over a sixteen-year period. J Thorac Cardiovasc Surg. 1979;78:365-82.

2. Motoyoshi N, Moizumi Y, Komatsu T, Tabayashi K. Intramural hematoma and dissection involving ascending aorta: the clinical features and prognosis. Eur J Cardiothorac Surg. 2003;24:237-42.

3. Attenhofer CH, Vogt PR, von Segesser LK, Dirsch OR, Ritter M, Jenni R. Leaking giant aneurysm of the aortic root due to cystic medial necrosis with pericardial tamponade mimicking type A aortic dissection. Thorac Cardiovasc Surg. 1996;44:103-4.

4. Isselbacher EM, Cigarroa JE, Eagle KA. Cardiac tamponade complicating proximal aortic dissection. Is pericardiocentesis harmful? Circulation. 1994;90:2375-8. 